\title{
Unrelenting Ocular Pain as a Masquerading Symptom of Occult Choroidal Metastasis
}

\author{
Jordan D. Deaner Renelle Pointdujour-Lim Emil Anthony T. Say Carol L. Shields \\ Ocular Oncology Service, Wills Eye Hospital, Thomas Jefferson University, Philadelphia, Pa., USA
}

\section{Key Words}

Choroidal metastasis · Lung carcinoma - Ocular pain .

Scleritis

\begin{abstract}
Purpose: To report a case of chronic eye pain as a presenting feature of choroidal metastasis from lung cancer. Methods: We report the case of a 58-year-old Caucasian woman with stage IV lung adenocarcinoma presenting with an 8-month history of left eye pain and blurred vision. Results: The patient had previously consulted 14 ophthalmologists with varying diagnoses including posterior scleritis and trigeminal neuralgia. Visual acuity at presentation was 20/20 in the right eye and 20/80 in the left eye. Examination of the right eye was normal, while the left eye showed ill-defined flat yellow discoloration of the choroid with overlying shifting subretinal fluid. Ultrasonography demonstrated a dense choroidal thickening measuring $2.6 \mathrm{~mm}$ in size and showing subretinal fluid. Enhanced depth imaging optical coherence tomography revealed choroidal thickening with a 'lumpy bumpy' surface topography consistent with a metastatic choroidal tumor presumably from the patient's lung adenocarcinoma. Fine needle aspiration biopsy followed by treatment was recommended, but the patient declined and later succumbed to metastatic disease. Conclusion: We present a
\end{abstract}

\section{KARGER}

(c) 2016 S. Karger AG, Basel

E-Mail karger@karger.com

www.karger.com/oop case of chronic eye pain associated with diffuse choroidal thickening from metastatic lung adenocarcinoma that was previously unrecognized and misdiagnosed. This case emphasizes the importance of recognizing pain as a presenting symptom of choroidal metastasis.

(c) 2016 S. Karger AG, Basel

Ocular metastases are generally symptomatic [1]. Of 950 metastatic uveal tumors in 520 eyes managed at an ocular oncology referral center, the most common ocular symptom at diagnosis included blurred vision (70\%), flashes and floaters (12\%), and less commonly pain (7\%) [1].

Ocular pain carries a broad differential diagnosis from dry eye to corneal abrasion, orbital pseudotumor, lacrimal gland malignancy, and many others. The combination of ocular pain with an intraocular mass narrows the differential to entities including scleritis, inflammatory retinal/choroidal mass with uveitis, hemorrhagic choroidal detachment, secondary glaucoma with retinal detachment from underlying retinal or choroidal tumor, choroidal melanoma with tumor necrosis, choroidal metastasis, and others. In an analysis of 4,940 patients with primary choroidal melanoma, Rishi et al. [2] found ocular pain as the main presenting feature in 15 cases $(<1 \%)$. They at- 
Fig. 1. a-d A 58-year-old Caucasian woman presented with an 8-month history of unrelenting eye pain, requiring narcotic medications, and blurred vision of the left eye. a Composite fundus photograph demonstrating an inferior retinal detachment with an ill-defined yellow discoloration in the macular region with retinal pigment epithelium clumping and loss. b Ultrasonography showing a slight thickening to the posterior globe and shallow inferior retinal detachment. c Enhanced depth imaging optical coherence tomography through the macula revealing ill-defined choroidal thickening with a 'lumpy bumpy' surface, loss of choroidal vascular structures, and shallow overlying subretinal fluid. d This 'lumpy bumpy' surface was more pronounced in the vertical cut.
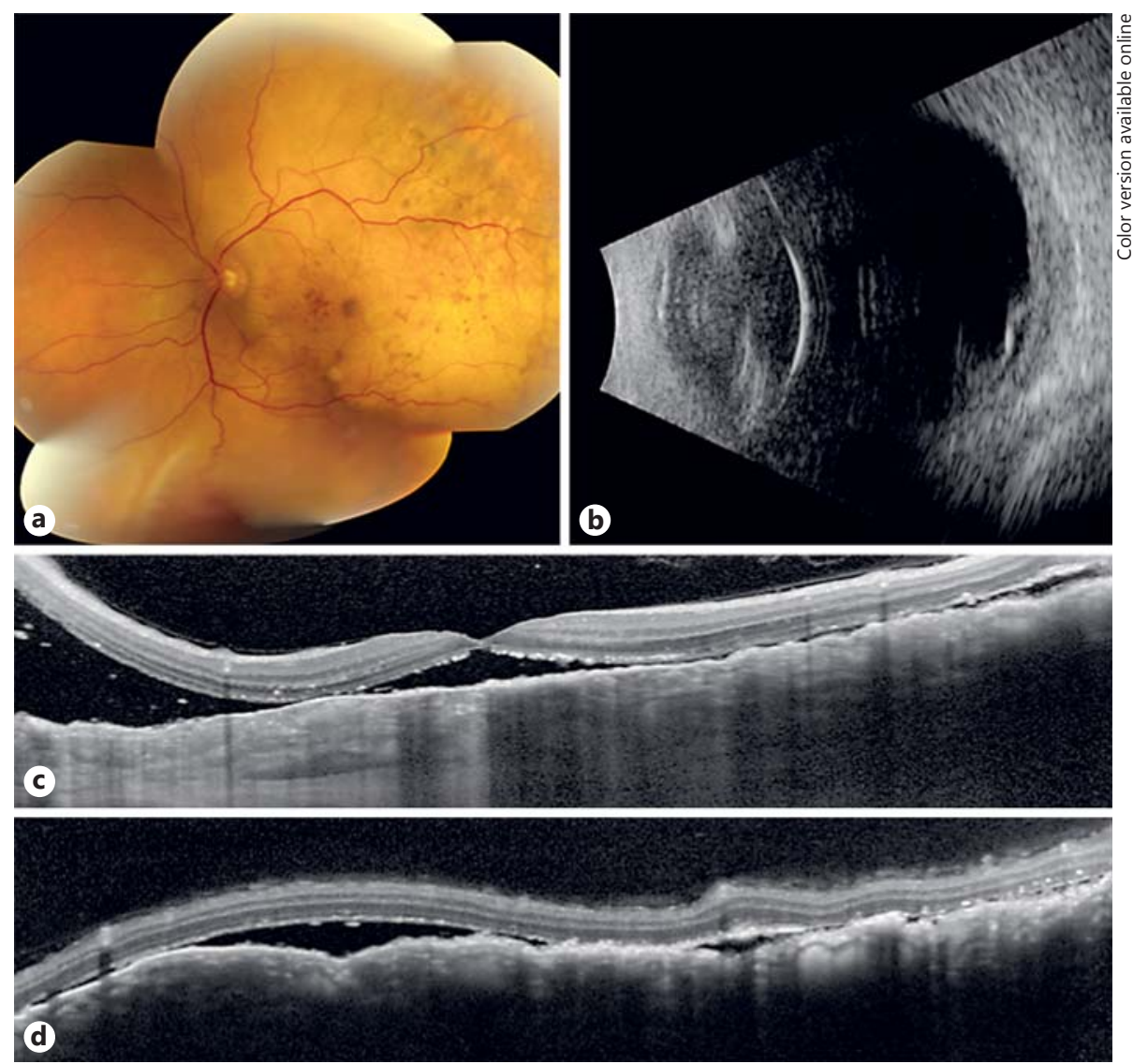

tributed the pain to glaucoma (neovascular or angle closure) or spontaneous tumor necrosis [2]. With regard to choroidal metastasis, low-grade ocular discomfort (pain) without glaucoma or visible necrosis has been recorded as a presenting symptom in $7 \%$ of cases overall, but increases to $13 \%$ in eyes with metastatic lung cancer [1]. The cause for metastasis-related pain is speculated to be related to tumor invasion, particularly within the sclera $[1,3]$.

Herein, we describe a patient with 8 months of severe, unrelenting periocular pain requiring narcotic analgesics. This patient was examined by multiple physicians who suspected scleritis. Ultimately, it was found that her pain was secondary to occult (flat) choroidal metastasis from lung adenocarcinoma.

\section{Case Report}

A 58-year-old Caucasian woman presented with an 8-month history of severe pain in the left eye and periocular region that radiated to the left temple and was associated with blurred vision. Her past medical history revealed a diagnosis of stage IV lung ad- enocarcinoma 5 years earlier with involvement of the opposite lung and lymph nodes, but without distant metastatic disease. Positron emission tomography-computed tomography (PET-CT) confirmed no evidence of distant metastasis and no sign of ocular metastasis. She was a nonsmoker and had previously declined suggested chemotherapy for prevention of metastatic disease. Regarding her eye symptoms, she had previously consulted 14 ophthalmologists who provided presumptive diagnoses of chronic posterior scleritis or trigeminal neuralgia. Medications for pain management included hydrocodone/acetaminophen 10/300 mg every $4-6 \mathrm{~h}$ with minimal relief. She declined treatment with corticosteroids.

On our examination, visual acuity was $20 / 20$ in the right eye and 20/80 in the left eye. Intraocular pressure and anterior segment findings were normal in each eye. There was no anterior segment inflammation or tumor. There were no signs of vitreous inflammation. The right fundus was normal.

The left fundus demonstrated bullous, shifting serous retinal detachment inferiorly without obvious tumor. However, there was ill-defined, patchy, flat yellow discoloration to the choroid with islands of retinal pigment epithelium clumping (fig. 1a). Ultrasonography confirmed inferior retinal detachment and a flat posterior globe echodensity measuring $2.6 \mathrm{~mm}$ in maximal thickness (fig. 1b). On enhanced depth imaging optical coherence tomography (EDI-OCT), the thickening involved the choroid and demonstrated a 'lumpy bumpy' surface topography, with overlying sub- 
retinal fluid and loss of normal choroidal vascular structures (fig. 1c, d). These features were suggestive of diffuse choroidal metastasis from previously documented lung cancer. Fine needle aspiration biopsy followed by external beam radiotherapy was advised, but she declined intervention and did not return for followup. Four months later, the patient succumbed from extensive disseminated systemic metastasis.

\section{Discussion}

Ocular pain is a common feature of several acute eye diseases and thus is a frequent primary complaint among patients presenting to the ophthalmologist [4]. Generating a differential diagnosis for any patient with ocular pain begins by identifying signs that specifically narrow the diagnosis such as an inflamed or 'red eye' [5-7] which suggests pathology of the anterior segment, ocular surface, or ocular adnexa such as a stye, chalazion, dry eye syndrome, corneal abrasion, conjunctivitis, scleritis, glaucoma, uveitis, and orbital inflammation [5-7]. In contrast, pain associated with a 'quiet [nonred] eye' requires the ophthalmologist to search out hidden signs of ocular pathology through a more detailed exam to reveal etiologies such as trigeminal neuralgia, posterior scleritis, optic neuritis, or intraocular tumors [5-7].

Pain with a quiet eye is a rare symptom of both primary and metastatic choroidal tumors [1-3]. Rishi et al. [2] described the classic presentation of ocular melanoma as a painless pigmented uveal mass with common symptoms including decreased visual acuity, flashes and floaters, and visual field defect. However, they identified 15 patients $(<1 \%)$ of a series of nearly 5,000 patients with uveal melanoma who presented with the main symptom of eye pain or headache [2]. In contrast, the complaint of eye pain is somewhat more prevalent with metastatic cancer. The most common presenting symptoms of ocular metastasis include blurred vision (70\%), flashes and floaters (12\%), pain (7\%), and no complaint (11\%) [1]. Shields et al. [1] found that the symptom of pain is disproportionately higher with metastatic lung cancer (13\%). Shah et al. [3] evaluated 194 patients with uveal metastasis from lung cancer and found the symptom of pain to be similarly relatively high (14\%). We suspect that the etiology of pain in this case was related to tumor necrosis, microscopic scleral invasion producing a scleritis-like picture, or perineural invasion around the posterior ciliary nerves $[1,3]$.

Our patient manifested an atypical presentation of a flat, diffuse, ill-defined, yellow choroidal abnormality without clinically evident mass, uncharacteristic of metastasis. The presence of shifting subretinal fluid and barely thickened choroid on OCT was suggestive of a possible metastasis [1]. Most metastatic lung cancers to the choroid are amelanotic (99\%) with a plateau (55\%) or dome (43\%) configuration [1]. This atypical flat, diffuse infiltration made the tumor more difficult to visualize ophthalmoscopically, possibly leading to its misidentification as an inflammatory condition, labeled as posterior scleritis [8]. Our case illustrates the importance of ocular pain and the need for a thorough evaluation in patients, especially those with a prior history of cancer. In these instances, imaging tests can help differentiate causes of eye pain.

By ultrasonography, choroidal metastases are acoustically dense with associated subretinal fluid, in contrast to choroidal melanoma which are generally hollow [9]. Posterior scleritis typically shows a 'T-sign' from accumulation of fluid in the sub-Tenon's space [10]. By EDI-OCT, these tumors can show different features. Choroidal metastasis generally demonstrates a 'lumpy bumpy' surface contour, often with overlying subretinal fluid and loss of normal choroidal structures [11]. In contrast, choroidal melanoma demonstrates a smooth, dome-shaped elevation with subretinal fluid and posterior scleritis can show choroidal thickening often with choroidal folds and without apparent loss of choroidal vascular spaces $[1,2,12$, 13]. When the clinical diagnosis is equivocal, even with appropriate imaging, fine needle aspiration biopsy can be revealing [1].

In summary, we presented a case of chronic ocular pain associated with an ill-defined posterior segment thickening that was found to be a metastatic choroidal tumor from lung adenocarcinoma, previously mistaken as posterior scleritis for nearly 1 year. This emphasizes the need for a thorough examination in patients with a history of cancer, and recognizing that pain can be the presenting symptom of metastasis.

\section{Conclusion}

A case of diffuse choroidal metastasis from lung adenocarcinoma with chronic unrelenting eye pain, previously unrecognized by a number of ophthalmologists before referral, emphasizes the importance of knowing that eye pain can be a presenting symptom of metastatic choroidal tumors.
58

Ocul Oncol Pathol 2017;3:56-59 DOI: $10.1159 / 000448720$
Deaner/Pointdujour-Lim/Say/Shields 


\section{Acknowledgement}

Financial support was provided by the Eye Tumor Research Foundation, Philadelphia, Pa., USA (C.L.S.). The funders had no role in the design and conduct of the study, in the collection, analysis and interpretation of the data, and in the preparation, review or approval of the manuscript. Carol L. Shields, MD, has had full access to all data in the study and takes responsibility for the integrity of the data and the accuracy of the data analysis.

\section{Statement of Ethics}

Our case report complies with all guidelines for human studies and animal welfare regulations. The patient has given informed consent. Our institute's committee on human research has approved the study's protocol. Our case study does not include any animal experimentation.

\section{Disclosure Statement}

No conflicting relationship exists for any author.

\section{References}

1 Shields CL, Shields JA, Gross NE, Schwartz GP, Lally SE: Survey of 520 eyes with uveal metastases. Ophthalmology 1997;104:12651276.

2 Rishi P, Shields CL, Khan MA, Patrick K, Shields JA: Headache or eye pain as the presenting feature of uveal melanoma. Ophthalmology 2013;120:1946-1947.

3 Shah SU, Mashayekhi A, Shields CL, Walia HS, Hubbard GB, Zhang J, Shields JA: Uveal metastasis from lung cancer: clinical features, treatment, and outcome in 194 patients. Ophthalmology 2014;121:352-357.

4 Roberts CJ, Macleod JD, Elkington AR: Ocular pain: a casualty study. The spectrum of pain in acute eye disease. Eye 1997;11:342344.
5 Waldman CW, Waldman SD, Waldman RA: Pain of the ocular and periocular region. Med Clin N Am 2013;97:293-307.

6 Lee AG, Beaver HA, Brazis PW: Painful ophthalmologic disorders and eye pain for the neurologist. Neurol Clin 2004;22:75-97.

7 Lee AG, Al-Zubidi N, Beaver HA, Brazis PW: An update on eye pain for the neurologist. Neurol Clin 2014;32:489-505.

8 McCluskey PJ, Watson PG, Lightman S, Haybittle J, Restori M, Branley M: Posterior scleritis: Clinical features, systemic associations, and outcome in a large series of patients. Ophthalmology 1999;106:2380-2386.

9 Sobattka B, Kreissig I: Ultrasonography of metastases and melanomas of the choroid. Current Opin Ophthalmol 1999;10:164-167.
10 Biswas J, Mittal S, Ganesh S, Shetty N, Gopal L: Posterior scleritis: clinical profile and imaging characteristics. Indian $\mathrm{J}$ Ophthalmol 1998;46:195-202.

11 Shields CL, Pellegrini M, Ferenczy SR, Shields JA: Enhanced depth imaging optical coherence tomography (EDI-OCT) of intraocular tumors: from placid to seasick to rock and rolling topography - the 2013 Francesco Orzalesi Lecture. Retina 2014;34:1495-1512.

12 Uchihori H, Nakai K, Ikuno Y, Gomi F, Hashida N, Jo Y, Nishida K: Choroidal observations in posterior scleritis using high-penetration optical coherence tomography. Int Ophthalmol 2014;34:937-943.

13 Taki W, Keino H, Watanabe T, Okada AA: Enhanced depth imaging optical coherence tomography of the choroid in recurrent unilateral posterior scleritis. Graef Arch Clin Exp 2013;251:1003-1004. 